\title{
SOLUCIÓN DE PROBLEMAS DE PRODUCCIÓN EN UNA EMPRESA MANUFACTURERA DE CALZADO EN LEÓN GUANAJUATO, MÉXICO
}

\author{
Production problems solution of a shoe manufacturing company in Leon Guanajuato, \\ México
}

\author{
Christian Arturo Quiroga Juárez \\ Ingeniero en Administración Industrial. Universidad de Guanajuato. Guanajuato, México. \\ christianquiroga87@hotmail.com \\ Jefferson Joao Aguirre Ramírez \\ M.Sc. en Ingeniería Administrativa. Universidad Nacional de Colombia. Medellín, Colombia. \\ ing.joao@gmail.com
}

\begin{abstract}
Cómo citar/ How to cite
Quiroga, C. y Aguirre, J. (2015). Solución de problemas de producción en una empresa de manufactura de suelas en León, Guanajuato, México. Revista CEA, 1(1), 121-130.
\end{abstract}

Recibido: 21 de mayo de 2014

Aceptado: 19 de agosto de 2014

\section{Resumen}

Este estudio se realizó de julio a noviembre de 2011 y tiene por objetivo identificar soluciones mediante procedimientos científicos a problemas de producción, presentados en una empresa dedicada a la fabricación de suelas para calzado, ubicada en León Guanajuato, México, mediante el diagnóstico y uso de algunas técnicas de ingeniería de métodos, estándares, diseño y simplificación del trabajo. Se utilizaron el diagrama de Ishikawa (Ishikawa, 1997) y el gráfico de Pareto (Niebel y Freivalds, 2004) para determinar y analizar las posibles causas generadoras de efectos negativos en la producción. Posteriormente, se obtuvieron los datos de entrada utilizando diagramas de operación y de flujo; como siguiente paso, se procedió a balancear las líneas de producción y ajustar las cargas de trabajo.

Los principales resultados del este estudio son el balanceo de líneas y el ajuste de cargas de trabajo, derivados de un estudio de tiempos y movimientos para incrementar la productividad de la empresa mediante la implementación de cambios en los métodos de trabajo; otro resultado fue la eliminación de movimientos innecesarios mediante un estudio de movimientos, incrementando la productividad al 56\% en una estación de trabajo. Se concluye que al implementar y dar mantenimiento a esta propuesta se mejorara la productividad de la empresa en cuestión.

Palabras clave: ingeniería de métodos, estándares, balanceo de líneas, diseño del trabajo, competitividad

\begin{abstract}
The objective of this case study is to identify solutions to problems through scientific procedures presented in a production company focused on the manufacture of shoe soles, located in Leon Guanajuato, Mexico, through the diagnosis and use of some of the engineering techniques methods, standards, job design and work simplification. The Ishikawa diagram and Pareto chart were used to identify and analyze the possible root causes of negative effects affecting the company production. Subsequently, the necessary data were obtained for this study using flow and operation diagrams. In the next stage, production lines were balanced and workloads were adjusted.

The main results of this study were to be able to identify specific solutions to increase business productivity by implementing some changes in the working methods and the use of appropriate tools. An increase of $56 \%$ was achieved in productivity in a workstation. Therefore, it is concluded that with proper implementation and monitoring of this proposal, productivity and efficiency in this footwear company can be improved.
\end{abstract}

Keywords: Engineering methods, standards, line balancing, job design and competitiveness. 


\section{INTRODUCCIÓN}

El objetivo de este caso de estudio es mejorar los procesos de planeación y control de la producción en una empresa dedicada a la producción de suelas, elaboración de suajes, fabricación y ventas de pegamento en la industria del calzado en León, Guanajuato, México. Para dimensionar la importancia de la industria del calzado, León Guanajuato -México- tiene una característica peculiar, ya que es aquí donde se concentra la mayor cantidad de empresas fabricantes de calzado a nivel estatal y a nivel país. Guanajuato es el primer lugar en producción de calzado seguido por el Distrito Federal y el estado de Jalisco, y en estas tres zonas de México se manufactura el $90 \%$ de la producción nacional (Ortiz y Martínez, 2000).

Debido a la magnitud de la industria del calzado en el estado de Guanajuato, la Cámara de la Industria del Calzado de este estado (CICEG) promueve acciones para mejorar los niveles de competitividad de la industria de calzado y fomenta la mejora en los procesos productivos, así como el avance en todas sus áreas funcionales (Cervantes y De La Garza, 2014). Con base en estas recomendaciones de la CICEG, las cuales se han venido promoviendo desde hace varios años, según palabras del director general de la empresa, la organización en cuestión buscó la mejor forma de hacer eficientes sus procesos productivos, y derivado de esa situación surge esta investigación orientada a la mejora de los procesos productivos.

Esta empresa contaba hasta la fecha en que se realizó este estudio con doce años de experiencia y con 22 empleados, de los cuales 9 rolaban 3 turnos de trabajo, es decir, la empresa estaba en producción 24 horas del día, y sus clientes principales se encuentran localizados en la ciudad de León.
Este estudio se llevó a cabo debido a que el gerente general, como ya se ha mencionado, había identificado la necesidad de volver eficientes los procesos de producción para cumplir en tiempo y forma con los requerimientos de los clientes, así como para disminuir los costos derivados de cuellos de botella y desperdicios excesivos de material. La metodología utilizada para abordar este caso de estudio fue derivada y adaptada del proceso sistemático para desarrollar un centro de trabajo, fabricar un producto o proporcionar un servicio (Niebel, Freivalds, y Niebel, 1999). La observación directa en la planta productiva se llevó a cabo durante los diferentes días de la semana, a distintas horas del día y sin repetir operarios, esto con la finalidad de considerar el cansancio físico por el horario, el clima y la edad del trabador.

Las conclusiones a las que se llegó se relacionan con la posibilidad de equilibrar la carga de las estaciones de trabajo con base en el balanceo de líneas; también se concluyó, con base en el estudio de tiempos y movimientos, que se podían crear células de trabajo, las cuales disminuirían los tiempos de producción. Con base en el estudio del lugar de trabajo, en una estación destinada para este, se determinó eliminar algunas operaciones manuales repetitivas con lo que se aumentó la productividad en más de un $50 \%$ y se estableció un sistema de reciclaje de material; esto se determinó con base en la experiencia de los operarios. La principal limitante fue la falta de concientización al personal acerca de la importancia de un estudio de esta magnitud, de esto se derivó cierto desinterés y miedo por parte de los operarios al momento de estudiar el proceso de producción de la empresa. 


\section{MARCO TEÓRICO}

\section{Historia}

Para conocer las bases de este estudio de caso es recomendable hacer un breve recorrido histórico. En 1776 Adam Smith en su obra La riqueza de las naciones propone la división del trabajo en tareas repetitivas y esto aumenta la producción de bienes (Smith, 1776). Otro suceso importante fue la Revolución Industrial previa al siglo XX, en esta época se llevó a cabo un cambio en los métodos de trabajo, se pasa de una economía rudimentaria a una economía industrializada, donde se desarrollaron invenciones de máquinas que con su potencia sustituyeron la fuerza humana, de esta forma se multiplicaba notablemente la producción y eficiencia en el trabajo.

En 1911 Frederick W. Taylor publica su obra titulada Principios de administración científica, la cual fue bien recibida por los gerentes deseosos de mayor productividad alrededor del mundo (Niebel y Freivalds, 2006). Esta obra ha sido la base para un sin número de estudios basados en su filosofía de la eficiencia y productividad. Frederick Taylor implementó un sistema de estudio del lugar del trabajo, así como el uso y determinación de estándares de trabajo (Taylor, 1911). El éxito de su administración científica fue tal, que se difundió por Estados Unidos, Francia Rusia, Japón, y en la actualidad lleva consigo el título de "Padre de la administración Científica (Rodríguez, 2006).

Los esposos Frank y Lilian Gilbreth fueron reconocidos como los primeros investigadores en estudiar los movimientos del cuerpo humano trabajando, ellos grabaron en una película los movimientos corporales con un aparato llamado microcronómetro, con el cual determinaban los movimientos del trabajador y cuánto duraban. El resultado de este estudio son los
17 movimientos manuales básicos Therbligs (Niebel y Freivalds, 1999).

Otro de los grandes aportes a la administración fue el de Henry Fayol, quien desarrolló su trabajo con base en la gerencia. Mientras Taylor trabajaba en la administración científica, Fayol se enfocó a la cabeza de la organización, y su investigación dio por resultado el proceso administrativo y los 14 puntos de administración, mismos que aún se siguen aplicando en varias organizaciones. Por otro lado, los aportes de Shingo, (1988) a la ingeniería industrial son de sustancial importancia para la compañía Toyota, ya que fue aquí donde desarrolló sus investigaciones y propuso las técnicas de SMED, para el control de calidad con el uso de Poka Yoke.

Otro de los grandes aportes en la ingeniería y administración de la producción se dio en cuanto a métodos cuantitativos o investigación de operaciones. Estos métodos tienen su origen en la milicia, cuando en la Segunda Guerra Mundial se convocó a una élite del saber para resolver problemas de asignación de recursos militares y su optimización mediante métodos estadísticos y matemáticos; este método es actualmente conocido como investigación de operaciones y se le han dado diversas aplicaciones hasta la fecha, por ejemplo: teoría de colas aplicada al transporte, teoría de redes aplicada al transporte terrestre y aéreo (en particular en el caso de la empresa Fedex), y teoría de los inventarios en las empresas que manejan almacenes, entre otros (Afanador y Ramos, 2013).

Al implementar las técnicas estadísticas y desarrollar las bases de los sistemas de control y planificación de la producción, independientemente del tamaño de la organización el objetivo que se busca es la eficiencia en la productividad (Chapman, 2006), de aquí la necesidad de utilizar las herramientas de la ingeniería industrial, en 
particular las técnicas de métodos de trabajo, establecimiento de estándares y diseño o rediseño del puesto de trabajo. Los aportes mencionados son solo parte de las herramientas que se pueden utilizar en un problema de producción y podrían usarse como una combinación de ellas, dependiendo del problema y de la creatividad del encargado de realizar el estudio y proponer soluciones a un problema.

En busca de mejorar los sistemas de producción y aumentar la productividad, y según las fuentes bibliográficas, se han realizado trabajos en diferentes áreas, tales como el trabajo realizado en G.E. Mustill (GEM) Company Ltda., donde se propuso el uso de la metodología de costeo basado en actividades $(A B C)$ con el fin de mejorar el rendimiento operativo (Gunasekaran y Singh, 1999); en la investigación bibliográfica se encontró otro trabajo de investigación enfocado a la mejora de procesos, específicamente en medir la madurez del sistema de producción en las empresas y posterior a esto proponer estrategias de mejora (Sommerville y Ransom, 2005).

En la literatura, cuando se mencionan temas relacionados con incrementar la eficiencia en procesos de producción, se entiende que hay que mejorar en todos los aspectos relacionados, y esto incluye cambios en los métodos de producción, más aún si se trata de favorecer al medio ambiente. Tal es el caso de la propuesta basada en cambiar el sistema de curtido de cuero "al cromo" por un sistema de curtido "al titanio», lo cual disminuye notablemente el grado de contaminación del medio ambiente y mejora las condiciones de trabajo del operador, ya que no es tóxico y no es alérgico (Ferrer, Riquelme, Segarra, Galiana y Navarro, 2012).

\section{Casos de referencia}

Uno de los casos más notables de aplicación de ingeniería de métodos de trabajo es el caso de Toyota, empresa asiática que ha heredado a la gestión de empresas herramientas que en la actualidad son aplicadas a nivel internacional. En este sentido, cabe destacar los sistemas justo a tiempo, el Kanban, así como los estándares flexibles de tiempo. Esta empresa se ha caracterizado por sus políticas innovadoras en la organización (Shingo, 1989).

Los sistemas de producción requieren cumplir con estándares de calidad, seguridad industrial, eficiencia y productividad. Es por esto que mayor cantidad de empresas vigilan y controlan sus procesos y ponen mayor atención al control del mantenimiento de la maquinaria, así como en las paradas de producción por cuellos de botella, los cuales representan altos costos de producción (Di Orio, Cândido, Barata, Scholze, Kotte, y Stokic, 2013).

Los problemas en producción, según la bibliografía revisada, son problemas a los que se enfrentan a diario los gerentes de producción, y la empresa en cuestión no es la excepción, ya que según el gerente general de la empresa, en el año 2012 se encontraban con problemas de productividad en la línea de producción de suelas, que incluye desperdicios de materia prima en el almacén así como en las máquinas de inyección de suela y desbalance en la línea de producción; estos problemas se hicieron evidentes al aplicar el gráfico de Pareto. 


\section{METODOLOGÍA}

Este estudio de carácter cuantitativo se realizó durante el periodo de julio a noviembre de 2011, en la planta de producción de una empresa dedicada a la manufactura de calzados, mediante la observación directa en el área de producción. La metodología usada para abordar este caso de estudio fue derivada y adaptada del proceso sistemático para desarrollar un centro de trabajo, fabricar un producto o proporcionar un servicio (Niebel et al., 1999).

- Definir cuáles eran las áreas donde se tenían los mayores problemas; para determinarlas se usaron los gráficos de Ishikawa y de Pareto.

- Obtener datos de ingeniería, de manufactura y costos, construcción de diagramas de flujo y de procesos.

Para la recolección de muestras de datos de manufactura se consideró el cansancio físico por el horario, el clima y la edad del trabador, por lo que se tomaron mediciones a diferentes días y horas, así como a distintos operarios que realizaban la misma operación.

La obtención de datos referentes a toma de tiempos y movimientos, se realizó con base en el número de observaciones sugeridas por la compañía General Electric (Niebel \& Freivalds, 2004), estas mediciones se llevaron a cabo por un periodo de 6 semanas y durante 4 horas diarias.
- El siguiente paso fue analizar los datos de "entrada» basado en los 9 enfoques principales para el análisis de la operación (Niebel et al., 1999).

- Posterior al análisis de los datos de «entrada», se diseñó una reestructuración al sistema de producción, basada en mejorar los diagramas de flujo y de operaciones, simplificación de las tareas, uso de las herramientas correctas, así como la definición de algunos tiempos estándar y el balanceo de la línea de producción.

- La siguiente etapa fue la implementación del método de producción reestructurado.

- La etapa final fue la evaluación del método propuesto.

\section{RESULTADOS}

Con base en el diagrama de Ishikawa, y derivado del análisis en conjunto con el gerente general y el jefe de producción, se llegó a la conclusión de que las áreas donde se desarrollaba la mayor cantidad de causas que producían un efecto negativo en la productividad fue la línea de acabado, que es donde se pintan las suelas, se recorta el exceso de material, se lava y se empaca para su comercialización.

También se obtuvieron las tablas de tiempos estándar producto del estudio de tiempos y movimientos para el área de "acabo», así como el índice de eficiencia de la línea de producción, como se muestra en la siguiente Tabla 1. 
Tabla 1. Estudio de tiempos estilo de suela 2361

Table 1. Time Study sole style 2361

\begin{tabular}{|c|c|c|c|}
\hline Actividad & Tiempo en minutos (ciclo) & Estándar de pares por hora & Horas por mil pares \\
\hline Rebabear & 0.3115 & 192.6163724 & 5.191666667 \\
\hline Matizar & 0.1109 & 541.0279531 & 1.848333333 \\
\hline Hacer pares & 0.06077 & 987.3292743 & 1.012833333 \\
\hline Embolsar & 0.03597 & 1668.056714 & 0.5995 \\
\hline Llevar a matizar & 0.0021065 & 28483.26608 & 0.035108333 \\
\hline & & & 8.687441667 \\
\hline Horas necesarias & Al $100 \%$ & Eficiencia del $75 \%$ & \\
\hline Estilo 2361 & 8.6874 horas & 11.5832 & \\
\hline
\end{tabular}

Fuente: Elaboración propia con base en Niebel y Freivalds (2006) y datos de la empresa

La Tabla 1 representa el primer esfuerzo por definir estándares con los cuales poder comparar, si después de implantar el método el proceso mejora o no. Es importante recalcar que en la empresa no se contaba con estándares de tiempos, y solo con base en experiencia se tenía un aproximado de la capacidad de producción.

Tabla 2. Estudio de tiempos estilo de suela 2882

Table 2. Time study sole style 2882

\begin{tabular}{|c|c|c|c|}
\hline Operación & $\begin{array}{l}\text { Tiempo } \\
\text { estándar }\end{array}$ & Estándar de pares & $\begin{array}{c}\text { Horas por mil } \\
\text { pares }\end{array}$ \\
\hline Rebabear y hacer pares & 0.5765 & 104.0763226 & 9.608333333 \\
\hline Matizar e inspeccionar suela & 0.0575 & 1043.478261 & 0.958333333 \\
\hline Hacer pares e inspeccionar suela & 0.06875 & 872.7272727 & 1.145833333 \\
\hline Embolsar & 0.1205 & 497.9253112 & 2.008333333 \\
\hline Llevar a matizar & 0.0021065 & 28483.26608 & 0.035108333 \\
\hline & & & 13.75594167 \\
\hline Urbán 2882 & Al $100 \%$ & Eficiencia de $75 \%$ & \\
\hline Horas para producir 1000 pares & 13.75 horas & 18.33333333 & \\
\hline
\end{tabular}

Fuente: Elaboración propia con base en Niebel y Freivalds (2006) y observación de la empresa

La Tabla 2 contiene los tiempos de una línea de suela llamada Urban 2882, la cual representó la mayor cantidad de observaciones, ya que al momento de matizar (acción mediante la cual se le da un acabado o se pinta la suela de acuerdo con las especificaciones del cliente) se realizaba mediante dos métodos totalmente diferentes, así que fue necesario observarlos a ambos, decidir cuál era el adecuado y trabajar con ese método, de esta manera continuar con la observación de tiempos y movimientos. 
Tabla 3. Estudio de tiempos estilo de suela 5772

Table 3. Time study sole style 5772

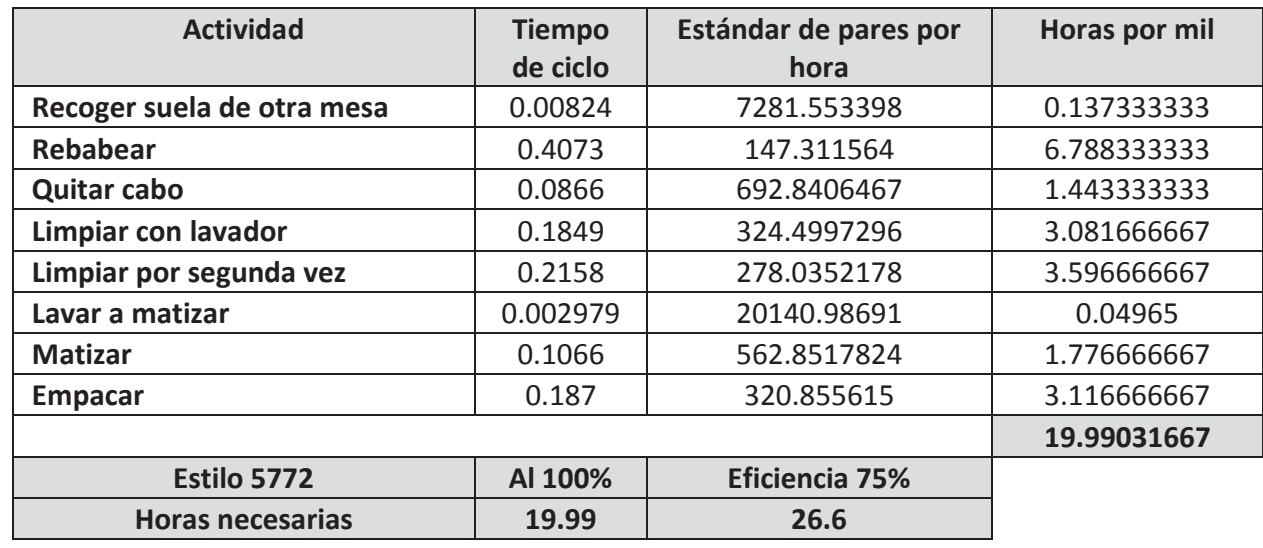

Fuente: Elaboración propia con base en Niebel y Freivalds (2006) y observación de la empresa

La importancia de la Tabla 3 recae en que esta línea de suela llamada 5772 fue la que contaba con mayor cantidad de demanda por un periodo de aproximadamente tres meses, y se consideró que se debían optimizar los tiempos y movimientos a la mayor brevedad, así como determinar sus estándares, ya que de hacerlo bien y con el mejor método representaría ganancias monetarias para la empresa, además de que esta línea dio la oportunidad de observar el método con que la realizaban por un mayor tiempo.
En la Tabla 4, la columna Valor $\mathrm{R}$, es el ritmo de la planta, considerando la mayor cantidad de producción requerida y los tiempos disponibles, así como la mayor cantidad de observaciones posibles para tener la mayor confiabilidad en la determinación de estándares. Adicionalmente, la cifra 2.8560 representa el número de operarios necesarios para realizar esas actividades. Es importante mencionar que había cinco operarios trabajando en una actividad que solo requería 2.85, o en términos reales, 3 operarios.

Tabla 4. Estudio de tiempos estilo de suela 5548

Table 4.Time study sole style 5548

\begin{tabular}{|c|c|c|c|c|c|}
\hline Operación & $\begin{array}{l}\text { Tiempo } \\
\text { estándar }\end{array}$ & Pares por hora & Horas por mil & Valor $\mathbf{R}$ & Operarios \\
\hline Rebabear & 0.2123 & 282.6189355 & 3.538333333 & 0.4831 & 0.585938 \\
\hline Quitar cabo & 0.061 & 983.6065574 & 1.016666667 & 0.4831 & 0.1683571 \\
\hline $\begin{array}{l}\text { Rebabear segunda } \\
\text { vez }\end{array}$ & 0.4556 & 131.6944688 & 7.593333333 & 0.4831 & 1.2574346 \\
\hline Llevar a matizar & 0.003125 & 19200 & 0.052083333 & 0.4831 & 0.0086249 \\
\hline Lavar & 0.1415 & 424.0282686 & 2.358333333 & 0.4831 & 0.3905334 \\
\hline Matizar & 0.0517 & 1160.541586 & 0.861666667 & 0.4831 & 0.1426896 \\
\hline Embolsar & 0.1096 & 547.4452555 & 1.826666667 & 0.4831 & 0.3024909 \\
\hline & & & & & 2.8560684 \\
\hline Estilo 5548 & $\begin{array}{c}\text { Horas al } \\
100 \%\end{array}$ & Eficiencia $75 \%$ & & & \\
\hline Horas /1000 prs. & 17.21 & 22.9 & & & \\
\hline
\end{tabular}

Fuente: Elaboración propia con base en Niebel y Freivalds (2006) observación de la empresa 
Tabla 5. Balanceo de líneas

Table 5. Balancing lines

\begin{tabular}{|l|c|c|c|}
\hline \multicolumn{4}{|c|}{ Considerando una tolerancia del 18.5\% } \\
\hline Operación & Tiempo estándar & Tolerancia 18.5\% & Número de operarios \\
\hline Rebabear & 0.2123 & 0.2515755 & 0.539206784 \\
\hline Quitar cabo & 0.061 & 0.072285 & 0.154929882 \\
\hline Rebabear segunda vez & 0.4556 & 0.539886 & 0.157148427 \\
\hline Llevar a matizar & 0.003125 & 0.003703125 & 0.007936982 \\
\hline Lavar & 0.1415 & 0.1676775 & 0.359386528 \\
\hline Matizar & 0.0517 & 0.0612645 & 0.131309424 \\
\hline Embolsar & 0.1096 & 0.129876 & 0.27836582 \\
\hline
\end{tabular}

Fuente: Elaboración propia con base en Niebel y Freivalds (2006) observación de la empresa

Después de hacer el estudio de tiempos y movimientos, se balanceó la línea de producción con base en el programa maestro de producción para determinar el número de operarios requeridos, así como el análisis de cargas de trabajo en cada estación como se ve en la Tabla 5.

Tabla 6. Ajuste de cargas de trabajo

Tabla 6. Adjusting workloads

\begin{tabular}{|c|c|c|c|c|c|c|}
\hline Operación & Pares/hora & $\begin{array}{c}\text { Tiempo } \\
\text { estándar }\end{array}$ & $\begin{array}{c}\text { Número de } \\
\text { operadores }\end{array}$ & $\begin{array}{c}\text { Número de } \\
\text { operadores }\end{array}$ & $\begin{array}{c}\text { Tiempo } \\
\text { medio de } \\
\text { ciclo }\end{array}$ & \% carga \\
\hline Rebabear & 217.0570679 & 0.276425 & 0.762972675 & 1 & 0.276425 & $60.67 \%$ \\
\hline $\begin{array}{c}\text { Rebabear } \\
\text { segunda vez }\end{array}$ & 131.6944688 & 0.4556 & 1.2527521391 & 1 & 0.4556 & $100.00 \%$ \\
\hline $\begin{array}{c}\text { Matizar, } \\
\text { lavary } \\
\text { embolsar }\end{array}$ & 198.1505945 & 0.3028 & 0.83577146 & 1 & 0.3028 & $66.46 \%$ \\
\hline
\end{tabular}

Con dos operarios del $100 \%$

\begin{tabular}{|c|c|c|c|c|c|c|}
\hline Operación & Pares/hora & $\begin{array}{c}\text { Tiempo } \\
\text { estándar }\end{array}$ & $\begin{array}{c}\text { Número de } \\
\text { operadores }\end{array}$ & $\begin{array}{c}\text { Número de } \\
\text { operadores }\end{array}$ & $\begin{array}{c}\text { Tiempo } \\
\text { medio de } \\
\text { ciclo }\end{array}$ & \% carga \\
\hline Rebabear & 217.0570679 & 0.276425 & 0.762972675 & 1 & 0.276425 & $91.29 \%$ \\
\hline $\begin{array}{c}\text { Rebabear } \\
\text { segunda } \\
\text { vez }\end{array}$ & 131.6944689 & 0.4556 & 1.257521391 & 2 & 0.2278 & $75.23 \%$ \\
\hline $\begin{array}{c}\text { Matizar, } \\
\text { lavar y } \\
\text { embolsar }\end{array}$ & 198.150594 & 0.3028 & 0.83577146 & 1 & 0.3028 & $100.00 \%$ \\
\hline
\end{tabular}

Fuente: Elaboración propia con base en Niebel y Freivalds (2006) observación de la empresa

En la Tabla 6 se observa una de las posibles combinaciones para disminuir la carga de la estación del 100\%. Se determinó que se necesitaban tan solo 4 personas para disminuir la carga de la estación del 100\%, y una de ellas podía ayudar a los demás 
alternadamente, y así disminuir los tiempos de producción.

Otros resultados referentes al desperdicio de materia prima fueron que algunos de los operarios no estaban concientizados de las pérdidas monetarias que produce el desperdicio injustificado de material, llegando a desperdiciar hasta $100 \mathrm{~kg}$ de materia prima por turno de ocho horas. Por su parte, gran cantidad de material desperdiciado en el almacén de materia prima provenía de falta de capacitación en los encargados de éste.

\section{CONCLUSIONES}

Con base en los resultados del estudio de tiempos y movimientos se propuso utilizar a uno de los operarios que contaban con mayor tiempo ocioso en la planta de producción y utilizarlo para equilibrar la carga de la estación del $100 \%$.

Otra propuesta con base en los resultados fue crear "células de trabajo», es decir, hacer dos equipos de dos personas cada uno encargados de atender una máquina de inyección de suela, debido a que dos personas en el departamento de acabado eran suficientes para atender a un solo operador de máquina de inyección de suela.

En lo referente al desperdicio de materia prima, se convocó a una reunión con los operarios donde se hizo notar que los desperdicios repercutían en la economía, no solo de la empresa sino de todo el personal, porque se veía reflejado en las utilidades que se dejaban de generar.

En relación al estudio de movimientos, eliminando operaciones repetitivas y movimientos innecesarios se consiguió que en una estación de pintado de suela se aumentara la productividad de un estilo en particular de suela, tal incremento fue de un $56 \%$ de productividad por hora.
Un resultado no esperado y muy productivo fue derivado de la charla de concientización del personal; un operario sugirió que en algunos materiales se podía reciclar el material proveniente de suelas en mal estado, mezclando aproximadamente un $30 \%$ de material reciclado y $70 \%$ de material de "primera mano». Aunado a esto, con base en prueba y error, se determinó que la cantidad aceptable de material de reciclaje que se podía mezclar era del $38 \%$, con este reciclaje en las primeras 3 semanas se logró ahorrar aproximadamente 5.550,0 kg de material.

Este análisis de ingeniería de métodos, estándares de trabajo y diseño del lugar de trabajo está limitado a la empresa en cuestión, sin embargo la metodología podría aplicarse con algunas adaptaciones a cualquier tipo de empresa, arrojando resultados beneficiosos y quizás algunos sorpresivos en beneficio de la productividad.

\section{REFERENCIAS}

Afanador, C., \& Ramos, C. (2013). PCA: Una nueva técnica de investigación de operaciones. México, Tesis de Licenciatura no publicada, Universidad de Guadalajara.

Cervantes, A., \& De La Garza, M. (2014). Prioridades y capacidades competitivas en organizaciones de manufactura de calzado en León, Guanajuato, México. Tópicos Selectos de Recursos, pp.103.

Chapman, S. N. (2006). Planificación y control de la producción. México: Pearson Educación.

Di Orio, G., Cândido, G., Barata, J., Scholze, S., Kotte, O., \& Stokic, D. (2013). Self-Learning Production Systems (SLPS) - Optimization of manufacturing process parameters 
for the shoe industry. In IEEE International Conference on Industrial Informatics (INDIN) (pp. 386-391). doi:10.1109/INDIN.2013.6622915

Ferrer, J., Riquelme, M. E., Segarra, V., Galiana, M. V., \& Navarro, S. (2012). Titanium-Tanned leather. In Proceedings of the 4th International Conference on Advanced Materials and Systems, ICAMS 2012 (pp. 543547).

Gunasekaran, A., \& Singh, D. (1999). Design of activity-based costing in a small company: a case study. Computers \& Industrial Engineering, 37(1), 413416.

Ishikawa, K. (1997). Qué es el control total de calidad: la modalidad japonesa. Colombia: Editorial Norma.

Niebel, B. W., \& Freivalds, A. (2004). Ingeniería industrial (11th ed.). México: Alfaomega.
Niebel, B. W., Freivalds, A., \& Niebel, B. W. (1999). Methods, standards, and work design. Boston, MA: McGrawHill

Ortiz, A., \& Martínez, A. (2000). Factores de competitividad, situación nacional y cadena productiva de la industria del calzado en León, Guanajuato. Economía, Sociedad y Territorio, 2(7), 533-568.

Shingo, S. (1988). Non-stock production: The Shingo system of continuous improvement. United States of America: Productivity Press.

Shingo, S. (1989). A study of the Toyota production system: From an Industrial Engineering Viewpoint. United States of America: Productivity Press.

Sommerville, I., \& Ransom, J. (2005). An empirical study of industrial requirements engineering process assessment and improvement. ACM Transactions on Software Engineering and Methodology (TOSEM), 14(1), 85-117. 